\title{
Diabetic Retinopathy and Diabetic Macular Edema
}

\author{
Steven R. Cohen • Thomas W. Gardner \\ Department of Ophthalmology and Visual Sciences, University of Michigan, Ann Arbor, MI, USA
}

\begin{abstract}
Diabetic retinopathy and diabetic macular edema result from chronic damage to the neurovascular structures of the retina. The pathophysiology of retinal damage remains uncertain but includes metabolic and neuroinflammatory insults. These mechanisms are addressed by intensive metabolic control of the systemic disease and by the use of ocular anti-inflammatory agents, including vascular endothelial growth factor inhibitors and corticosteroids. Improved understanding of the ocular and systemic mechanisms that underlie diabetic retinopathy will lead to improved means to diagnose and treat retinopathy and better maintain vision. @ 2016 S. Karger AG, Basel
\end{abstract}

This chapter summarizes the pathogenesis, risk factors, diagnosis, signs and symptoms, and treatment options for diabetic retinopathy (DR) and diabetic macular edema (DME). The complex nature of DR has led to a variety of therapies, but treatments for DR and DME are still challenging, particularly in the stages when retinopathy is mild and patients retain good vision.

\section{Diabetic Retinopathy Prevalence}

DR is one of the major complications of diabetes and is a leading cause of blindness and vision impairment. Approximately $75 \%$ of persons suffer- ing from type 1 diabetes develop retinopathy, while approximately $50 \%$ of persons with type 2 diabetes may develop retinopathy [1], and approximately $25 \%$ of persons with diabetes may develop macular edema. During the next two decades, over 360 million people worldwide are projected to have diabetes and its complications [2]. Fortunately, the prevalence of severe retinopathy and nephropathy in patients with type 1 diabetes has diminished over the past 35 years due to improved medical care [3], but the recent epidemic of type 2 diabetes requires a new understanding of the biology of DR and our approach to its prevention and treatment. Approximately 500,000 persons in the United States have clinically significant DME, with an annual incidence of 75,000 , and approximately 700,000 have proliferative DR, with an annual incidence of 65,000 [4].

\section{Risk Factors}

The clinical risk factors for DR have long been recognized to include diabetes severity and duration, hypertension, presence of other complications, anemia, hyperlipidemia, insulin resistance and deficiency, and a family history of DR (reviewed by Antonetti et al. [5] and Girach and Vignati [6]). While the benefits of decreasing $\mathrm{HbA}_{1 \mathrm{c}}$ or blood pressure levels have been demonstrated 
in large clinical trials $[7,8]$, there is presently no integrated index of the risk of DR and other complications that include these known variables. Such an integrated index would greatly facilitate the identification of patients who are at increased risk of complications and who merit aggressive systemic and ocular therapy. A study to identify risk factors associated with the progression to proliferative $\mathrm{DR}(\mathrm{PDR})$ identified $\mathrm{Hb}_{1 \mathrm{c}}$, diabetic nephropathy, and nonhealing foot ulcers as three risk factors that can help predict progression to PDR. From this, the authors derived a risk score [9]. However, most of the risk for retinopathy is not accounted for by traditional clinical indices [10].

Indeed, much work remains to be done to determine the potential contribution of genetic factors and the impact of systemic inflammation. A single nucleotide polymorphism in the promoter region of the erythropoietin gene confers a twofold increased risk of PDR and end-stage renal disease versus patients without this mutation [11]. This finding was the result of large-scale genomic screening in three population groups and demonstrates the power of interdisciplinary collaborative studies. However, genetic studies have yet to yield major insights into DR, partly related to the complexity of the disease and the small size of most studies [12].

Several studies have demonstrated a relationship between plasma levels of inflammatory markers and DR [13-16], but it remains unclear if these inflammatory molecules contribute directly to the retinal damage in diabetes or simply reflect the systemic inflammatory state, and if they predict progression of disease or can be employed as markers of treatment response.

\section{Etiology and Pathogenesis}

The etiology and pathogenesis of DR and DME have been the subject of much research and debate for the past three decades. At present, the un- derstanding of these processes is undergoing a dramatic shift from a strictly vascular focus to a more comprehensive view of the disease.

The vascular lesions of DR have been emphasized from the first case report of DR in 1855, reviewed by Wolfensberger and Hamilton [17], through the development of fluorescein angiography and trypsin digest studies of retinal blood vessels in the early 1960s, and in the classification of DR for the laser treatment trials in the 1970s and 1980s. The microvascular disease approach led to successful development of laser photocoagulation for proliferative retinopathy and DME. Expanded research into molecular pathways and chemical mediators has now led to the use of pharmacologic treatments for DR and DME in addition to laser therapy.

Concurrent with the development of fluorescein angiography and trypsin digest studies, two studies of postmortem human eyes in patients with DR $[18,19]$ emphasized the degeneration of the retinal neuropil even in regions remote from sites of vascular lesions. Electroretinographic studies subsequently confirmed the impairment in neural retinal function in patients with DR [20-23], indicating the presence of Müller cell defects and predicting the risk of progression from severe nonproliferative to high-risk proliferative retinopathy. However, a lack of appreciation of the biology of the transparent neurosensory retina by clinicians may have limited the clinical application of these findings.

Over the past decade, numerous studies of animals and humans have confirmed that all retinal cell types are damaged by diabetes, including loss of inner retinal neurons and their projections [24, 25], dysfunction of Müller cells [26-28] and astrocytes $[26,29]$, activation of microglia, resident immune cells of the nervous system [30,31], and degeneration of the pigment epithelium [32]. Thus, the current biological definition of DR includes all functional and structural changes in the retina due to diabetes, and the term 'microvascular disease' does not fully describe the retinal 
features of diabetes and is misleading in its limited scope.

The interrelationships between these retinal cell changes remain to be resolved. Do the vascular changes cause neuronal or glial cell defects, or vice versa, or are all the changes part of a coordinated sequential process? This question remains difficult to resolve.

Neuroretinal cell changes have not been well appreciated because they are invisible to clinical examination by ophthalmoscopy, fluorescein angiography, and standard optical coherence tomography (OCT). Therefore, new diagnostic methods based on changes in retinal neural cells are now being developed, as discussed below. In eyes with cystoid macular edema, the clinical appearance of cysts and corresponding leakage of fluorescein dye suggest that leakage of the perifoveal capillaries likely accounts for macular thickening. However, histologic studies show loss of retinal neurons in addition to foveal cysts; a reduction in macular edema may not result in visual improvement if retinal neurons are nonfunctional. Hence, recognizing both the clinical and biological aspects of retinal changes in DME and DR are essential to understanding the mechanisms of vision loss and designing appropriate treatments.

The cell biology of DR and DME is now becoming better understood, but the pathophysiologic processes that lead to the cellular lesions remain highly controversial. Hence, there is currently no well-established unifying hypothesis that accounts for the full range of vascular and neural changes, and impairment in visual function. The major concepts are summarized as follows.

First, the basic pathophysiology of diabetes itself deserves emphasis. Diabetes mellitus refers to 'honey urine' and reflects the emphasis on glucose accumulation. Clinicians diagnose diabetes based on elevated blood glucose levels because blood glucose concentration correlates closely with clinical manifestations and is readily mea- sured. However, both type 1 and type 2 diabetes results from impaired insulin action, which secondarily leads to hyperglycemia, hyperlipidemia, and elevated serum branched-chain amino acids, with catabolic degradation of tissues such as skeletal muscle and adipose tissues.

The role of excess glucose in DR has received extensive investigation as part of the aldose reductase (polyol) pathway, nonenzymatic glycation and advanced glycation end product receptor activation, protein kinase $\mathrm{C} \beta$ activation, and oxidative and nitrative stress. Most of these changes have been investigated in rodent models of diabetes, where they seem to have a close connection with vascular changes. However, the promise of aldose reductase inhibitors in diabetic rats was not borne out in diabetic dogs or humans [33]. Likewise, advanced glycation end product blockers did not demonstrate beneficial effects on diabetic complications in humans and were toxic. The only successful treatment based on a glucoseactivated pathway has been the protein kinase $C \beta$ inhibitor, ruboxistaurin, which has been shown to reduce vision loss in DR [34-36]. However, this medication failed to achieve Food and Drug Administration approval and did not achieve statistical significance in the most recent studies [37].

Inflammation is an early and intrinsic feature of systemic insulin resistance and diabetes that involves the release of cytokines from adipose tissue that impairs insulin action, and affects patients with both type 1 and type 2 diabetes [38, 39]. Interestingly, DR was formerly termed 'diabetic retinitis' [40] before the inflammatory component was understood. The clinical picture of DR provides numerous clues to the presence of chronic inflammation, including diffuse vascular dilation, tortuosity, leakage and neovascularization, atrophy of the retinal neural parenchyma, edema of the macula, and eventual fibrosis. Cellular studies reveal activation of microglia [2931] and leukostasis [41]. Together, these clinical and laboratory-based studies leave little doubt as 
to the presence of inflammation in DR, even though it is much more insidious than the uveitis associated with other systemic conditions such as sarcoidosis or multiple sclerosis.

The relationship of systemic inflammation to $\mathrm{DR}$ is currently unclear. Inflammatory responses are part of the intrinsic immune system reaction to multiple stresses, such as injury, ischemia, and infection, and inflammation is designed to limit the stress. Unrelenting stress and inflammation, however, lead to the clinical features associated with tissue damage that we recognize as 'disease'. If the intrinsic immune response in diabetes is similar to that in other tissues, its activation in diabetes may be designed to maintain cell viability in the presence of metabolic stress. The retina may respond with increased production of growth factors such as vascular endothelial growth factor (VEGF) and erythropoietin, cytokines, complement, and microglial cell activation as a physiologic compensatory response. If the metabolic stress is relieved, the inflammation may subside, but prolonged metabolic stress may lead to a maladaptive response in which inflammation would damage tissue because of tissue edema, invasion of circulating immune cells, and fibrosis. Thus, the inflammatory response is probably a doubleedged sword with both beneficial and detrimental aspects. At present, it is not clear which components of the inflammatory response are critical or which may serve as useful therapeutic targets.

Impaired insulin action is the sine qua non of diabetes and, along with glucagon excess, accounts for numerous tissue changes, including atrophy of skeletal muscles and adipose tissue. Until recently, there was very little information on the role of the insulin receptor signaling system in the retina. Biochemical studies show that the receptor is structurally similar to that in the liver, is expressed in all retinal cells, forms heterodimers with the insulin-like growth factor 1 receptor, and has high basal activity relative to other tissues such as the liver [42, 43]. In diabetic rodents, the basal activity decreases progressively with time, and systemic insulin treatment restores the activity [44]. The impaired activity may predispose the retina to additional metabolic insults from systemic inflammation and hypertension, which also incite inflammation via the retinal renin-angiotensin system [45]. This dynamically regulated insulin receptor system likely maintains the activity and viability of postmitotic retinal cells, but full understanding of this process is currently lacking.

In summary, the etiology of DR is tied closely with the fundamental processes of diabetes itself, but the precise mechanisms that initiate or perpetuate retinal damage and lead to vision impairment remain to be clarified.

\section{Diagnosis and Ancillary Testing/Differential Diagnosis}

DR is diagnosed clinically on the basis of visible hemorrhages, microaneurysms, cotton wool spots, lipid exudates, and neovascularization. Fundus photography and fluorescein angiography have greater sensitivity than ophthalmoscopy because of superior optics, the enhanced contrast of fluorescein angiography, confirmation of vascular leakage, and the ability of the observer to review magnified images without the interference of patients moving or blinking.

Prevention of vision loss requires better understanding of the fundamental processes that impair vision and improved diagnostic tests to provide parameters to gauge the response to pharmacologic interventions. Retinal dysfunction in diabetes has long been known to begin before the onset of microvascular lesions. Changes in color vision, contrast sensitivity, visual fields, and electroretinography responses have been documented thoroughly [23] over the past three decades. Technological advances have allowed newer imaging modalities to be applied to retinal diseases, both in clinical practice and research. Most prominent has been the increasing 
utilization of OCT for the detection and quantification of DME. Macular thickness maps and OCT images provide guidance for treatment response to anti-VEGF therapy and macular laser therapy, and have been extensively utilized in large clinical trials [46]. The high resolution available with spectral-domain OCT allows for close study of the retinal layers and cells in clinical disease [47]. Ultra-wide-field imaging is currently used for the screening and detection of DR as is ultra-wide-field angiography [48, 49]. Adaptive optics is being applied to in vivo retinal imaging, allowing visualization of individual retinal cells and microvasculature that may help clarify the structure and function of the retina in diabetes and its response to treatment $[50,51]$. Other functional tests have been investigated to detect early visual dysfunction in DR, such as the use of frequency doubling perimetry, which indicates inner retinal impairment as a significant contributor to visual dysfunction in DR [52]. However, no test of retinal function other than visual acuity has been shown conclusively to predict the onset or progression of retinopathy or vision loss, or to be a useful endpoint for clinical trials. Thus, the understanding of the biology of DR continues to evolve and provides new opportunities to move the timeline of diagnosis and treatment much earlier in the course of diabetes [53]. The process of translating the results of laboratory findings into clinical utility is complex, expensive, slow, and requires new ways of studying DR [54], but investments in the process should pay substantial dividends in the future.

\section{Signs and Symptoms}

$\mathrm{DR}$ is unusual among retinal diseases in that the symptoms vary widely and may be out of proportion to the severity of retinal pathology. For example, eyes with mild DME involving the fovea of one eye can have symptomatically decreased vision whereas eyes with severe proliferative reti- nopathy may have 20/20 acuity and no symptoms until they begin to develop vitreous hemorrhage. This variability and discrepancy between structure and function are the rationale for programs that screen populations at risk to identify and treat asymptomatic patients to reduce the risk of vision loss [55].

\section{Treatment Options}

The key to preventing and controlling ocular complications from diabetes is strict glycemic control and control of blood pressure. The importance of controlling the underlying disease process should be stressed to patients and has been proven in several large clinical trials $[7,56$, 57]. Once the patient presents with PDR, panretinal photocoagulation remains the standard treatment at present and significantly reduces the risk of vision loss $[58,59]$. Strong evidence also exists for use of focal macular laser photocoagulation in the prevention of vision loss secondary to DME $[58,60,61]$. For many years, these were the only interventions for which strong clinical evidence existed. With the use of anti-VEGF agents expanding beyond macular degeneration, more options have become available in preventing and even reversing vision loss secondary to DR and DME.

VEGF plays a critical role in DR and DME. Diabetes increases the production of VEGF, leading to increased vascular permeability and angiogenesis [62]. As a result, blockage of the VEGF-A molecule has been the recent focus of pharmacologic therapy aimed at treating these diseases [63]. In 2012, intravitreal ranibizumab received FDA approval for the treatment of DME based on the results of several large clinical trials [64-69]. These studies demonstrated that intravitreal ranibizumab significantly reduces macular edema and results in improved visual acuity for patients with DME. In some studies, ranibizumab was more effective than focal laser therapy at reducing 
DME, with laser therapy providing no additional benefit. The DRCR.net (Diabetic Retinopathy Clinical Research Network) Protocol I study evaluated ranibizumab with prompt or deferred fo$\mathrm{cal} /$ grid macular laser therapy for center-involving DME and demonstrated a clear benefit of using ranibizumab when initiating treatment. Longer-term results of this study also showed that adding macular laser therapy at the initiation of ranibizumab was no better, and may in fact be worse, compared with delaying laser therapy to $>24$ weeks $[65,66,68]$. READ-2 compared ranibizumab alone to laser alone or ranibizumab plus laser, and found mean best-corrected visual acuity (BCVA) to be better with ranibizumab monotherapy, with sustained efficacy [67]. RESTORE (Ranibizumab plus Macular Laser Photocoagulation) also demonstrated superiority of ranibizumab monotherapy over macular laser photocoagulation, and found no additional benefit of ranibizumab therapy combined with macular laser therapy [69]. The RISE and RIDE studies have provided additional evidence that ranibizumab monotherapy provides rapid and sustained results in improving macular edema and BCVA. In addition, a delay in the initiation of ranibizumab therapy resulted in limited improvement in BCVA in the 36-month results [64]. Bevacizumab is frequently used off-label to treat DME as well and has demonstrated efficacy in several clinical trials [70, 71]. BOLT (Bevacizumab or Laser Therapy) compared intravitreal bevacizumab to laser therapy alone and found mean BCVA to be significantly better in the bevacizumab group versus laser therapy alone [70]. Another anti-VEGF agent, aflibercept, received FDA approval in 2014 for the treatment of DME and has the potential to decrease the frequency of required injections [72]. The DRCR.net Protocol T study evaluated the relative efficacy and safety of aflibercept, bevacizumab, and ranibizumab for the treatment of center-involving DME [73]. The 1-year results concluded that all three agents improved vision in eyes with DME. In eyes with better baseline vi- sual acuity (Snellen 20/40 or better), there was no statistically significant difference among the three agents. In eyes with worse baseline visual acuity (Snellen 20/50 or worse), aflibercept achieved greater average gains in visual acuity compared to bevacizumab and ranibizumab, and this difference was statistically significant [73].

The use of anti-VEGF agents has allowed for a tremendous improvement in the clinician's ability to treat DME. However, the use of focal/grid macular laser therapy still plays a critical role in the treatment of this disease process and can help individualize treatments for patients who may not respond to or tolerate treatment with frequent anti-VEGF medications.

Panretinal photocoagulation remains the standard for treatment of PDR. However, given the prominent role of VEGF as a driving mediator in angiogenesis, studies are exploring the effects of intravitreal anti-VEGF therapy on DR and its possible role in treatment. In these studies, ranibizumab has been shown to reduce the probability of retinopathy progression in eyes with and without PDR [74]. Studies are ongoing in determining the utility of using anti-VEGF agents to delay panretinal photocoagulation for PDR (NCT01489189). Bevacizumab has also been incorporated pre- and postoperatively for use in vitrectomy for PDR. Studies have examined its effects on intraoperative maneuvers and intraoperative bleeding, as well as rates of postoperative vitreous hemorrhage [75]. However, high-quality evidence is still lacking in demonstrating a clear benefit.

Given that inflammation plays a critical role in DR and DME, various corticosteroids have been used in the treatment of DME. In addition to antiinflammatory effects of corticosteroids, they also inhibit VEGF and increase tight junctions between capillary endothelial cells [76]. Corticosteroids are effective in various forms and delivery systems when used in combination with macular laser therapy. Intravitreal triamcinolone combined with prompt focal/grid laser therapy has 
demonstrated equal efficacy in comparison to ranibizumab monotherapy in pseudophakic patients $[65,68]$. However, intravitreal triamcinolone as a monotherapy was less effective when compared to macular laser photocoagulation [77]. Evidence also exists that intravitreal triamcinolone may reduce the progression of PDR [74]. Other corticosteroids that have been used for DME in combination include sustained drug delivery implants for dexamethasone (Ozurdex) and fluocinolone acetonide (Retisert and Iluvien). These treatments are not without significant ocular side effects, which include an increased incidence of glaucoma and a high rate of cataract formation requiring cataract surgery $[78,79]$.

Surgery with vitrectomy remains an indispensable treatment option for patients with complications from DR including nonclearing vitreous hemorrhage and tractional retinal detachments. In addition, vitrectomy with induction of posterior vitreous detachment can be used for the treatment of DME with a tractional component $[80,81]$. This treatment option has led to interest in the use of enzymatic vitreolysis to treat DME [82], which may become more of a possibility given FDA approval of ocriplasmin for use in symptomatic vitreomacular adhesions [83]. Research is ongoing for additional pharmacotherapies that can modulate molecules other than VEGF that contribute to inflammation and vascular permeability, resulting in macular edema and angiogenesis [84].

\section{Treatment Outcomes and Prognosis}

All treatments for the established vascular lesions of $\mathrm{DR}$ reduce the risk of blindness and vision loss but seldom restore normal vision $[85,86]$. The continued impact of diabetes, hypertension, and other systemic insults continues to reduce vision. Long-term follow-up of patients treated with panretinal photocoagulation in the Diabetic Retinopathy Study retained useful vision that gradually declined over time [87]. With results of the clinical trials involving intravitreal anti-VEGF for DME, the treatment paradigm has shifted from preventing vision loss to actually improving vision in patients with DME [62]. Although these results are encouraging, treating the long-term effects of metabolic dysfunction on neuroretinal cells presents a challenge. Early disease detection and prevention through intensive blood glucose control remains essential in delaying the onset and progression of DR [88].

\section{Conclusion and Key Points}

Retinopathy continues to be a frightening and devastating consequence of diabetes for patients and their families. The prognosis for patients with diabetes and the ensuing complications improves with overall enhancements in diabetes management, but the ocular complications are still diagnosed and treated late in terms of the biological processes. Fundamentally different approaches to the problem must be taken as the number of persons with diabetes doubles over the next 25 years to prevent a global epidemic of preventable blindness. Intravitreal agents have added an invaluable management option in addition to laser photocoagulation and vitrectomy. However, given the high disease burden, the practicality of delivering this care in a timely and effective fashion remains a challenge, particularly in developing nations. This further stresses the importance of early disease detection and treatment through large-scale screening and patient education. Utilizing developments in technology and telemedicine will likely play a key role in achieving this goal.

On a more molecular level, research is needed to treat the metabolic pathway upstream, as opposed to treating the downstream consequences. Better information is needed on the mechanism of the effects of intensive insulin therapy and how to design and deliver agents to achieve the effect without inducing hypoglycemia or other adverse 
events. Viewing DR as a specific consequence of the metabolic derangement of diabetes provides promise that pharmacotherapy should have a dramatic impact on the risk of vision loss in persons with diabetes.

\section{Acknowledgment}

This work was supported by a Research to Prevent Blindness Physician-Scientist Award, the Taubman Medical Research Institute, JDRF, and R01EY20582.

\section{References}

1 The Eye Diseases Prevalence Research Group: The prevalence of diabetic retinopathy among adults in the United States. Arch Ophthalmol 2004;122:552563.

2 Wilkinson-Berka JL, Miller AG: Update on the treatment of diabetic retinopathy. ScientificWorldJournal 2008;8:98-120.

3 Hovind P, Tarnow L, Rossing K, et al: Decreasing incidence of severe diabetic microangiopathy in type 1 diabetes. Diabetes Care 2003;26:1258-1264.

4 Bhavsar AR, Drouilhet JH, Atebara NH, et al: Diabetic Retinopathy. 2014, http:// emedicine.medscape.com/ article/1225122-overview\#a0156.

5 Antonetti DA, Barber AJ, Bronson SK, et al: Diabetic retinopathy: seeing beyond glucose-induced microvascular disease. Diabetes 2006;55:2401-2411.

6 Girach A, Vignati L: Diabetic microvascular complications - can the presence of one predict the development of another? J Diabetes Complications 2006; 20:228-237.

7 The effect of intensive treatment of diabetes on the development and progression of long-term complications in insulin-dependent diabetes mellitus. The Diabetes Control and Complications Trial Research Group. N Engl J Med 1993;329:977-986.

8 Matthews DR, Stratton IM, Aldington SJ, Holman RR, Kohner EM, UK Prospective Diabetes Study Group: Risks of progression of retinopathy and vision loss related to tight blood pressure control in type 2 diabetes mellitus: UKPDS 69. Arch Ophthalmol 2004;122:1631-1640.

9 Harris Nwanyanwu K, Talwar N, Gardner TW, Wrobel JS, Herman WH, Stein JD: Predicting development of proliferative diabetic retinopathy. Diabetes Care 2013;36:1562-1568.

10 Antonetti DA, Klein R, Gardner TW: Diabetic retinopathy. N Engl J Med 2012;366:1227-1239.
11 Tong Z, Yang Z, Patel S, et al: Promoter polymorphism of the erythropoietin gene in severe diabetic eye and kidney complications. Proc Natl Acad Sci U S A 2008;105:6998-7003.

12 Kuo JZ, Wong TY, Rotter JI: Challenges in elucidating the genetics of diabetic retinopathy. JAMA Ophthalmol 2014; 132:96-107.

13 Meleth AD, Agron E, Chan CC, et al: Serum inflammatory markers in diabetic retinopathy. Invest Ophthalmol Vis Sci 2005;46:4295-4301.

14 Schram MT, Chaturvedi N, Schalkwijk CG, Fuller JH, Stehouwer CD, EURODIAB Prospective Complications Study Group: Markers of inflammation are cross-sectionally associated with microvascular complications and cardiovascular disease in type 1 diabetes the EURODIAB Prospective Complications Study. Diabetologia 2005; 48 : 370-378.

15 Nin JW, Ferreira I, Schalkwijk CG, et al: Levels of soluble receptor for AGE are cross-sectionally associated with cardiovascular disease in type 1 diabetes, and this association is partially mediated by endothelial and renal dysfunction and by low-grade inflammation: the EURODIAB Prospective Complications Study. Diabetologia 2009;52:705-714.

16 Zhou J, Wang S, Xia X: Role of intravitreal inflammatory cytokines and angiogenic factors in proliferative diabetic retinopathy. Curr Eye Res 2012;37:416-420.

17 Wolfensberger TJ, Hamilton AM: Diabetic retinopathy - an historical review. Semin Ophthalmol 2001;16:2-7.

18 Bloodworth JM Jr: Diabetic retinopathy. Diabetes 1962;11:1-22.

19 Wolter JR: Diabetic retinopathy. Am J Ophthalmol 1961;51:1123-1141.
20 Simonsen SE: ERG in juvenile diabetics: a prognostic study; in Goldberg MF, Fine SL (eds): Symposium on the Treatment of Diabetic Retinopathy. Arlington, US Department of Health, Education and Welfare,1969, pp 681-689.

21 Bresnick GH: Diabetic retinopathy viewed as a neurosensory disorder. Arch Ophthalmol 1986;104:989-990.

22 Bresnick GH, Palta M: Predicting progression to severe proliferative diabetic retinopathy. Arch Ophthalmol 1987;105: 810-814.

23 Ghirlanda G, Di Leo MA, Caputo S, Cercone S, Greco AV: From functional to microvascular abnormalities in early diabetic retinopathy. Diabetes Metab Rev 1997;13:15-35.

24 Barber AJ, Lieth E, Khin SA, Antonetti DA, Buchanan AG, Gardner TW: Neural apoptosis in the retina during experimental and human diabetes. Early onset and effect of insulin. J Clin Invest 1998; 102:783-791.

25 Gastinger MJ, Singh RS, Barber AJ: Loss of cholinergic and dopaminergic amacrine cells in streptozotocin-diabetic rat and Ins2Akita-diabetic mouse retinas. Invest Ophthalmol Vis Sci 2006;47: 3143-3150.

26 Barber AJ, Antonetti DA, Gardner TW: Altered expression of retinal occludin and glial fibrillary acidic protein in experimental diabetes. The Penn State Retina Research Group. Invest Ophthalmol Vis Sci 2000;41:3561-3568.

27 Mizutani M, Gerhardinger C, Lorenzi M: Muller cell changes in human diabetic retinopathy. Diabetes 1998;47:445-449.

28 Puro DG: Diabetes-induced dysfunction of retinal Müller cells. Trans Am Ophthalmol Soc 2002;100:339-352.

29 Rungger-Brandle E, Dosso AA, Leuenberger PM: Glial reactivity, an early feature of diabetic retinopathy. Invest Ophthalmol Vis Sci 2000;41:1971-1980. 
30 Krady JK, Basu A, Allen CM, et al: Minocycline reduces proinflammatory cytokine expression, microglial activation, and caspase- 3 activation in a rodent model of diabetic retinopathy. Diabetes 2005;54:1559-1565.

31 Zeng HY, Green WR, Tso MO: Microglial activation in human diabetic retinopathy. Arch Ophthalmol 2008;126: 227-232.

32 Bensaoula T, Ottlecz A: Biochemical and ultrastructural studies in the neural retina and retinal pigment epithelium of STZ-diabetic rats: effect of captopril. J Ocul Pharmacol Ther 2001;17:573-586.

33 A randomized trial of sorbinil, an aldose reductase inhibitor, in diabetic retinopathy. Sorbinil Retinopathy Trial Research Group. Arch Ophthalmol 1990;108: 1234-1244.

34 PKC-DRS Study Group: The effect of ruboxistaurin on visual loss in patients with moderately severe to very severe nonproliferative diabetic retinopathy: initial results of the Protein Kinase C $\beta$ Inhibitor Diabetic Retinopathy Study (PKC-DRS) multicenter randomized clinical trial. Diabetes 2005;54:2188-2197.

35 Group P-D, Aiello LP, Davis MD, et al: Effect of ruboxistaurin on visual loss in patients with diabetic retinopathy. Ophthalmology 2006;113:2221-2230.

36 Aiello LP, Vignati L, Sheetz MJ, et al: Oral protein kinase $C \beta$ inhibition using ruboxistaurin: efficacy, safety, and causes of vision loss among 813 patients (1,392 eyes) with diabetic retinopathy in the Protein Kinase C $\beta$ Inhibitor-Diabetic Retinopathy Study and the Protein Kinase C $\beta$ Inhibitor-Diabetic Retinopathy Study 2. Retina 2011;31:2084-2094.

37 Sheetz MJ, Aiello LP, Davis MD, et al: The effect of the oral PKC $\beta$ inhibitor ruboxistaurin on vision loss in two phase 3 studies. Invest Ophthalmol Vis Sci 2013;54:1750-1757.

38 Greenbaum CJ: Insulin resistance in type 1 diabetes. Diabetes Metab Res Rev 2002;18:192-200.

39 Wellen KE, Hotamisligil GS: Inflammation, stress, and diabetes. J Clin Invest 2005;115:1111-1119.

40 Dana GW: Type of diabetes mellitus associated with diabetic retinitis. AMA Arch Ophthalmol 1953;50:123-124.

41 Barouch FC, Miyamoto K, Allport JR, et al: Integrin-mediated neutrophil adhesion and retinal leukostasis in diabetes. Invest Ophthalmol Vis Sci 2000;41: 1153-1158.
42 Reiter CE, Sandirasegarane L, Wolpert $\mathrm{EB}$, et al: Characterization of insulin signaling in rat retina in vivo and ex vivo. Am J Physiol Endocrinol Metab 2003;285:E763-E774.

43 Reiter CE, Gardner TW: Functions of insulin and insulin receptor signaling in retina: possible implications for diabetic retinopathy. Prog Retin Eye Res 2003; 22:545-562.

44 Reiter CE, Wu X, Sandirasegarane L, et al: Diabetes reduces basal retinal insulin receptor signaling: reversal with systemic and local insulin. Diabetes 2006;55: 1148-1156.

45 Wilkinson-Berka JL: Diabetes and retinal vascular disorders: role of the reninangiotensin system. Expert Rev Mol Med 2004;6:1-18.

46 Virgili G, Menchini F, Murro V, Peluso E, Rosa F, Casazza G: Optical coherence tomography (OCT) for detection of macular oedema in patients with diabetic retinopathy. Cochrane Database Syst Rev 2011;7:CD008081.

47 Sim DA, Keane PA, Fung S, et al: Quantitative analysis of diabetic macular ischemia using optical coherence tomography. Invest Ophthalmol Vis Sci 2014;55:417-423.

48 Silva PS, Cavallerano JD, Sun JK, Noble J, Aiello LM, Aiello LP: Nonmydriatic ultrawide field retinal imaging compared with dilated standard 7-field 35mm photography and retinal specialist examination for evaluation of diabetic retinopathy. Am J Ophthalmol 2012; 154:549.e2-559.e2.

49 Wessel MM, Aaker GD, Parlitsis G, Cho M, D’Amico DJ, Kiss S: Ultra-wide-field angiography improves the detection and classification of diabetic retinopathy. Retina 2012;32:785-791.

50 Lombardo M, Parravano M, Serrao S, Ducoli P, Stirpe M, Lombardo G: Analysis of retinal capillaries in patients with type 1 diabetes and nonproliferative diabetic retinopathy using adaptive optics imaging. Retina 2013;33:1630-1639.

51 Dubow M, Pinhas A, Shah N, et al: Classification of human retinal microaneurysms using adaptive optics scanning light ophthalmoscope fluorescein angiography. Invest Ophthalmol Vis Sci 2014;55:1299-1309.

52 Jackson GR, Scott IU, Quillen DA, Walter LE, Gardner TW: Inner retinal visual dysfunction is a sensitive marker of non-proliferative diabetic retinopathy. Br J Ophthalmol 2012;96:699-703.
53 Field MG, Elner VM, Puro DG, et al: Rapid, noninvasive detection of diabetes-induced retinal metabolic stress. Arch Ophthalmol 2008;126:934-938.

54 Gardner TW, Jackson GR, Quillen DA, Scott IU: Diabetic retinopathy: translating discoveries to treatments; in Greenbaum C, Harrison LC (eds): Diabetes: Translating Discoveries into Practice. New York, Informa, 2008, pp 173-192.

55 Stefansson E, Bek T, Porta M, Larsen N, Kristinsson JK, Agardh E: Screening and prevention of diabetic blindness. Acta Ophthalmol Scand 2000;78:374-385.

56 Progression of retinopathy with intensive versus conventional treatment in the Diabetes Control and Complications Trial. Diabetes Control and Complications Trial Research Group. Ophthalmology 1995;102:647-661.

57 Stratton IM, Kohner EM, Aldington SJ, et al: UKPDS 50: risk factors for incidence and progression of retinopathy in type II diabetes over 6 years from diagnosis. Diabetologia 2001;44:156-163.

58 Photocoagulation treatment of proliferative diabetic retinopathy. Clinical application of Diabetic Retinopathy Study (DRS) findings, DRS Report Number 8. The Diabetic Retinopathy Study Research Group. Ophthalmology 1981;88:583-600.

59 Early photocoagulation for diabetic retinopathy. ETDRS report number 9. Early Treatment Diabetic Retinopathy Study Research Group. Ophthalmology 1991; 98:766-785.

60 Mohamed Q, Gillies MC, Wong TY: Management of diabetic retinopathy: a systematic review. JAMA 2007;298:902-916.

61 Photocoagulation for diabetic macular edema. Early Treatment Diabetic Retinopathy Study report number 1. Early Treatment Diabetic Retinopathy Study research group. Arch Ophthalmol 1985; 103:1796-1806.

62 Boyer DS, Hopkins JJ, Sorof J, Ehrlich JS: Anti-vascular endothelial growth factor therapy for diabetic macular edema. Ther Adv Endocrinol Metab 2013;4:151-169.

63 Miller JW, Le Couter J, Strauss EC, Ferrara N: Vascular endothelial growth factor $\mathrm{A}$ in intraocular vascular disease. Ophthalmology 2013;120:106-114.

64 Brown DM, Nguyen QD, Marcus DM, et al: Long-term outcomes of ranibizumab therapy for diabetic macular edema: the 36-month results from two phase III trials: RISE and RIDE. Ophthalmology 2013;120:2013-2022. 
65 Diabetic Retinopathy Clinical Research Network, Elman MJ, Aiello LP, et al: Randomized trial evaluating ranibizumab plus prompt or deferred laser or triamcinolone plus prompt laser for diabetic macular edema. Ophthalmology 2010;117:1064.e35-1077.e35.

66 Diabetic Retinopathy Clinical Research Network, Elman MJ, Qin H, et al: Intravitreal ranibizumab for diabetic macular edema with prompt versus deferred laser treatment: three-year randomized trial results. Ophthalmology 2012;119: 2312-2318.

67 Do DV, Nguyen QD, Khwaja AA, et al: Ranibizumab for Edema of the Macula in Diabetes study: 3-year outcomes and the need for prolonged frequent treatment. JAMA Ophthalmol 2013;131:139145.

68 Elman MJ, Bressler NM, Qin H, et al: Expanded 2-year follow-up of ranibizumab plus prompt or deferred laser or triamcinolone plus prompt laser for diabetic macular edema. Ophthalmology 2011;118:609-614.

69 Mitchell P, Bandello F, Schmidt-Erfurth U, et al: The RESTORE study: ranibizumab monotherapy or combined with laser versus laser monotherapy for diabetic macular edema. Ophthalmology 2011;118:615-625.

70 Rajendram R, Fraser-Bell S, Kaines A, et al: A 2-year prospective randomized controlled trial of intravitreal bevacizumab or laser therapy (BOLT) in the management of diabetic macular edema: 24-month data: report 3. Arch Ophthalmol 2012;130:972-979.

71 Nepomuceno AB, Takaki E, Paes de Almeida FP, et al: A prospective randomized trial of intravitreal bevacizumab versus ranibizumab for the management of diabetic macular edema. Am J Ophthalmol 2013;156:502.e2-510.e2.
72 Moradi A, Sepah YJ, Sadiq MA, et al: Vascular endothelial growth factor Trap-Eye (aflibercept) for the management of diabetic macular edema. World J Diabetes 2013;4:303-309.

73 Diabetic Retinopathy Clinical Research Network, Wells JA, Glassman AR, et al: Aflibercept, bevacizumab, or ranibizumab for diabetic macular edema. N Engl J Med 2015;372:1193-1203.

74 Bressler SB, Qin H, Melia M, et al: Exploratory analysis of the effect of intravitreal ranibizumab or triamcinolone on worsening of diabetic retinopathy in a randomized clinical trial. JAMA Ophthalmol 2013;131:1033-1040.

75 Ahn J, Woo SJ, Chung H, Park KH: The effect of adjunctive intravitreal bevacizumab for preventing postvitrectomy hemorrhage in proliferative diabetic retinopathy. Ophthalmology 2011;118: 2218-2226.

76 Gardner TW, Antonetti DA, Barber AJ, LaNoue KF, Levison SW: Diabetic retinopathy: more than meets the eye. Surv Ophthalmol 2002;47(suppl 2):S253S262.

77 Diabetic Retinopathy Clinical Research Network: A randomized trial comparing intravitreal triamcinolone acetonide and focal/grid photocoagulation for diabetic macular edema. Ophthalmology 2008; 115:1447-1449, 1449.e1-1449.e10.

78 Callanan DG, Gupta S, Boyer DS, et al: Dexamethasone intravitreal implant in combination with laser photocoagulation for the treatment of diffuse diabetic macular edema. Ophthalmology 2013; 120:1843-1851.

79 Campochiaro PA, Brown DM, Pearson $A$, et al: Sustained delivery fluocinolone acetonide vitreous inserts provide benefit for at least 3 years in patients with diabetic macular edema. Ophthalmology 2012;119:2125-2132.

80 Diabetic Retinopathy Clinical Research Network Writing Committee, Haller JA, Qin H, et al: Vitrectomy outcomes in eyes with diabetic macular edema and vitreomacular traction. Ophthalmology 2010;117:1087.e3-1093.e3.
81 Shamsi HN, Masaud JS, Ghazi NG: Diabetic macular edema: new promising therapies. World J Diabetes 2013;4:324338.

82 Diaz-Llopis M, Udaondo P, Millan JM, Arevalo JF: Enzymatic vitrectomy for diabetic retinopathy and diabetic macular edema. World J Diabetes 2013;4: 319-323.

83 Stalmans P, Benz MS, Gandorfer A, et al: Enzymatic vitreolysis with ocriplasmin for vitreomacular traction and macular holes. N Engl J Med 2012;367:606-615.

84 Gardner TW, Antonetti DA: Novel potential mechanisms for diabetic macular edema: leveraging new investigational approaches. Curr Diab Rep 2008;8:263269 .

85 Tonello M, Costa RA, Almeida FP, Barbosa JC, Scott IU, Jorge R: Panretinal photocoagulation versus PRP plus intravitreal bevacizumab for high-risk proliferative diabetic retinopathy (IBeHi study). Acta Ophthalmol 2008;86:385389.

86 Diabetic Retinopathy Clinical Research Network, Scott IU, Edwards AR, et al: A phase II randomized clinical trial of intravitreal bevacizumab for diabetic macular edema. Ophthalmology 2007;114: 1860-1867.

87 Blankenship GW: Fifteen-year argon laser and xenon photocoagulation visual results of Bascom Palmer Eye Institute's patients participating in the Diabetic Retinopathy Study. Trans Am Ophthalmol Soc 1990;88:179-185; discussion 85-89.

88 Aiello LP, DCCT/EDIC Research Group: Diabetic retinopathy and other ocular findings in the Diabetes Control and Complications Trial/Epidemiology of Diabetes Interventions and Complications study. Diabetes Care 2014;37:1723.
Thomas W. Gardner, MD, MS

Department of Ophthalmology and Visual Sciences

University of Michigan

Ann Arbor, Ml 48105 (USA)

E-Mail tomwgard@med.umich.edu 